\title{
Introducing Patient Reported Measures (PRMs) Into Finnish Public Maternity Service: A Study on Professionals' Views
}

\section{An Chen ( $\square$ an.chen@aalto.fi )}

Institute of Healthcare Engineering, Management and Architecture (HEMA), Department of Industrial Engineering and Management, Aalto University, Maarintie 8, 02150, Espoo, Finland

\section{Kirsi Väyrynen}

Central Finland Central Hospital

\section{Riikka-Leena Leskelä}

Nordic Healthcare Group Oy

\section{Seppo Heinonen}

Helsinki University Hospital

Paul Lillrank

Aalto University

\section{Aydin Tekay}

Helsinki University Hospital

\section{Paulus Torkki}

University of Helsinki

\section{Research Article}

Keywords: patient-reported measures (PRMs), patient-reported outcome measures (PROMs), patientreported experience measures (PREMs), health outcomes, maternity care, pregnancy, childbirth, implementation, qualitative research

Posted Date: January 11th, 2021

DOl: https://doi.org/10.21203/rs.3.rs-129756/v1

License: (c) (1) This work is licensed under a Creative Commons Attribution 4.0 International License. Read Full License 


\section{Abstract}

Background: The importance and potential benefits of introducing patient reported measures (PRMs) into health care service have been widely acknowledged, yet the experience regarding their implementation into practice is limited. There is a considerable paucity of research in adopting PRMs in maternity care. This study, which utilizes the PRMs included in Pregnancy and Childbirth (PCB) outcome set developed by International Consortium for Health Outcomes Measurement (ICHOM) as sample measures, aims to elicit Finnish professionals' views on PRMs and to explore the applicability of PRMs in Finnish public maternity care.

Methods: This qualitative study, applying semi-structured interviews, is designed to describe the local professionals' views towards the application of PRMs in Finnish public maternity care. Professionals were asked to assess the PRMs defined in ICHOM PCB set and provide their expectations and concerns on the implementation of PRMs in Finnish public maternity service.

Results: Twenty professionals participated in the interviews. Participants agreed on the importance and relevance of the PRMs questions included in ICHOM PCB set for delivering and developing maternity care in Finland. However, they criticized the number and length of questions as well as the recommended time points of data collection. In addition, for a successful implementation, various steps like developing suitable questions, redesigning service pathway and protocols, and motivating patients were considered to be important. Also, some potential obstacles, difficulties and risks associated with the implementation were underlined.

Conclusion: This study indicates that the implementation of PRMs into Finnish public maternity service is possible, highly relevant and important. However, the adoption of PRMs into routine practice may be challenging and will require a series of efforts. This study shows viewpoints from Finnish professionals who have not participated in developing the ICHOM PCB standard set and provides important insights on the development and implementation of PRMs.

\section{Introduction}

Patient-reported measures (PRMs) gather information about the outcomes and the experiences of health services as perceived and described by patients. PRMs help service providers understand what matters to patients, and they have become accepted as a core dimension of healthcare quality [1, 2]. Increasing the use of these measures and incorporating them into existing measurement dashboards are considered as effective strategies to achieve patient-centered care and value-based health care, a widely accepted healthcare delivery model introduced by Michael Porter [1, 3-8]. In PRMs, patient-reported outcome measures (PROMs) capture patients' perceptions of their health status and help evaluate the result of a clinical intervention, while patient-reported experience measures (PREMs) collect information on patients' personal experience of the healthcare services they have received [6]. By implementing PRMs to complement clinical outcome measures, healthcare providers can assess whether the clinical outcomes 
are relevant for the patient, holistically and longitudinally evaluate whether health care services have an effect on patients, and communicate better with patients [9-11]. With PRMs, the patient will be able to make informed decisions and assume some responsibility for the management of their health conditions [12]. For healthcare administrators, PRMs provide an opportunity to identify care gaps and understand vulnerable groups in healthcare [1]. While the importance and potential benefits of introducing PRMs into healthcare outcome measurement and evaluation have been widely acknowledged, knowledge and experience of integrating PRMs into routine practices and using them to improve healthcare delivery are limited [2]. Simple and occasional surveys on patient perception and satisfaction without standardized, systematic and comprehensive measures are inadequate for providers to capture the true feelings of patients or the gaps in the delivery of the services.

Pregnancy and childbirth are life-changing events for women and families, consisting of physical, psychological and emotional changes as well as personal experiences during the whole process [13] that PRMs could be applied to capture. So far, there is a lack of successful and transferable cases of adopting PRMs in maternity care. Finnish public maternity care system has obtained international acclaims for its lowest maternal mortality rate as well as for the equity and equality in distributing the services among women and their families. However, as the bar for quality raises, and patient-centered and value-based care become a megatrend, the evaluation of the quality of care requires new insights. Until now, Finland has not established well-structured and proper measures that can systematically reflect women's and their families' views and experiences on the process and outcomes of care they receive. The implementation of PRMs into Finnish public maternity care could solve these problems.

A variety of available methods, tools and instruments could potentially be helpful in developing PRMs to address general or specific issues across the pathway of pregnancy and childbirth [14-19]. A comprehensive and international standardized set of PROMs and PREMs for maternity care has been developed by the International Consortium on Health Outcome Measurement (ICHOM) [20], a not-for-profit organization that aims to facilitate the adoption of value-based health care worldwide. ICHOM published an outcomes and quality measurement set for pregnancy and childbirth (PCB set) in 2018. This set covers the full cycle of care with five critical data-collection time points and contains PROMs (e.g. health related quality of life, pain with intercourse, and postpartum depression) and PREMs (e.g. satisfaction with care, confidence in healthcare providers and confidence in healthcare providers) (Additional file 1 displays PROMs and PREMs and other patient reported items defined in ICHOM PCB set) [21]. Although ICHOM has provided a reference guide with hands-on materials [22] to facilitate the process of implementing this standard set into routine practice, a complete pre-implementation investigation of local acceptability and applicability is required. Until recently, we are aware of only few studies assessing the local implementation of the PRMs defined in ICHOM PCB outcome set [23-25]. The implementation of PRMs are affected by a wide range of factors, e.g. healthcare contexts, service settings, health conditions of populations, and local culture [26]. More knowledge and deeper insights for the implementation of PRMs in various settings are needed. This study, using PROMs and PREMs defined in ICHOM PCB set as sample measures, aims to elicit Finnish professionals' views on PRMs, to explore the applicability of 
PRMs for public maternity care in Finland, where a specific maternity care system has been developed, and to provide useful insights of integrating PRMs into health care pathways.

\section{Methods}

\section{Research contexts}

Finland provides mothers with free maternity care as part of the national health care and social welfare system [27]. The aim is to have equal and safe care available for all women during the course of pregnancy and childbirth [28]. In 2018, 47,913 children were born in Finland [29]. Over 99.5\% of all pregnant mothers in Finland seek care from public maternity service [28, 30]. Maternity care in Finland, funded by municipal and state tax revenues, is a mutual effort of authorities and multiple health care professionals and providers [28]. The Ministry of Social Affairs and Health is responsible for guiding the service provision and development. Municipalities or local authorities are responsible for basic services, providing community-based maternity and child health clinic (Neuvola) services as part of primary health care. Neuvola services, led by public health nurses (PHN) and medical doctors, include regular perinatal monitoring, health examinations at predetermined times, health counselling, and family support with attention to relationships and parenting. Hospital districts (20 in total) organize specialized medical care, offer screening and laboratory tests, and run delivery units in their hospitals. Midwives, working at maternity units of hospitals, are the main service providers for childbirth. National Institute for Health and Welfare of Finland (THL) organizes and controls birth registers and follows maternity-related statistics.

Figure 1 shows the general process of Finnish maternity care. The process begins when a pregnant woman contacts Neuvola and schedules the first appointment. Before or at the first appointment, essential family and health-related information is collected from the pregnant woman and her partner. The pregnant woman is recommended to meet her PHN minimum of eight times, and additionally a medical doctor at least once before birth. The PHN informs the pregnant woman about the availability of the free and voluntary prenatal screening and testing program with different alternatives, and schedules the screening appointments at hospital district screening units. In case of pregnancy complications, Neuvola refers mothers to antenatal clinics or emergency departments of the secondary care hospitals. Upon entering the third trimester, first-time parents can take part in family education and coaching sessions organized by municipalities. When the delivery begins, the mother calls an assigned delivery ward at the secondary care hospital. In the delivery room, midwives take care of the labor process, other professionals including obstetricians, anesthetists, and pediatricians participate when needed, and obstetricians oversee the delivery process. After the birth, the mother and the newborn are transferred to a postnatal unit staffed by midwives, registered nurses and obstetricians. After being discharged from the delivery hospital, mother meets the PHN at home or at a Neuvola clinic within a week for a postpartum check-up. In addition, there is an extensive doctor's checkup at Neuvola between two and four months after childbirth. 


\section{Semi-structured interview}

This qualitative study applied semi-structured interviews among local professionals in Finland to collect their views on the applicability of PRMs defined in the ICHOM PCB set for the Finnish context and their concerns on the implementation of PRMs measurement. The interview consisted of two parts applying different data collection techniques and analysis methods. In the first part, professionals were asked to evaluate PRMs defined in ICHOM PCB set in terms of importance of each measure, and appropriateness of the time points of measuring and reporting. Professionals gave scores of importance (0-Not important at all; 1-Nice to have; 2-Important) and appropriateness of the time points (0-Not appropriate at all; 1-To some extend; 2-Appropriate) and provided comments for each measure. In the second part, we explored professionals' views on the implementation of PRMs in Finnish public maternity services. The concrete interview questions of the second part were developed from Unified Theory of Acceptance and Use of Technology (UTAUT) model [31], which was tailored to this project with five constructs, including:

- Performance - whether an individual believes / expects that using the system will help to attain gains (i.e. expected usefulness)

- Effort - whether an individual believes / expects that there is any ease/complexity and cost associated with the use of the system

- Conditions - whether an individual believes /expects that there is any condition, e.g. organizational culture and technical infrastructure, existing to support/hinder the use of the system

- Perceived risk - whether an individual believes / expects that using the system takes a possibility that something unpleasant or unwelcome (harm or damage) will happen to the current system and status

- Social norms and influence - whether an individual believes that there is any social norm associated with the use of the system; whether an individual perceives that important others believe he or she should / should not use the system

\section{Recruitment}

Starting from September of 2019, local professionals, including obstetricians, midwives, public nurses, researchers, educators and administrators, who were working in the field of pregnancy and childbirth were approached by two interviewers. Purposive sampling was used as the technique to recruit participants and get a sample that could represent the population regarding the topic of this study. We assumed a certain degree of homogeneity shared by our targeted group of professionals and experts, and according to Guest, Bunce and Johnson (2006) we estimated that 15-20 in-depth interviews could be enough to draw some valid conclusions. In order to ensure adequate representativeness of the sample, we continued to conduct the interviews until we reached data saturation, and the additional data made little changes in analytic patterns and themes [32,33]. Professionals were informed about the purpose and protocol of the interview. All the informants participating in the interviews gave oral or written informed consent. 


\section{Data processing and analysis}

Interviews were tape recorded and transcribed (translated into English if the interview was conducted in Finnish), and imported into Microsoft Excel and Atlas.ti qualitative data analysis software (V.8.4). Multiple methods were used to study participants' views and gain detailed insights of the phenomena being explored. For part 1 of the interviews, assessing measures defined in ICHOM PCB set, we listed the scores and counted the number of professionals for each score. We read all comments given by professionals on each measure, put same and similar comments together, and identified representative viewpoints according to the number of professionals who mentioned them. For part 2 of the interviews, exploring professionals' views on the implementation of PRMs, we adopted a framework approach embedded with thematic analysis $[34,35]$. The primary themes were defined by the modified UTAUT model, which were used as a coding framework. Data were deductively coded and grouped into the predefined themes. Within each primary theme, codes were grouped and categorized to explore patterns and inductively develop sub-themes. Two researchers independently coded transcripts, identified the items relevant to each primary theme of the contextualized UTAUT model, and compared the coding to reach consensus on a coding scheme. Then the two researchers discussed, explored the relationship between the codes, reached an agreement on the prominent sub-themes within each pre-defined primary theme. We enhanced the validity of our analysis and findings by organizing discussions in the research team and getting feedback from some of the professionals we interviewed.

\section{Results}

\section{Basic characteristics of participants}

Altogether 20 professionals participated in interviews. Table 1 summarizes the basic characteristics of participants. Our sample covers the most important and relevant professionals from the main organizations across the maternity care network in Finland. Most interviews were conducted in English, and three in Finnish. The majority of the participants were from the capital region, i.e. Helsinki and Uusimaa hospital district (HUS), which is the biggest hospital district, with roughly $30 \%$ of all deliveries in Finland [29]. Each interview lasted from 1.5 to 2 hours.

\section{Assessment on PRMs defined in ICHOM PCB Set}

Table 2 summarizes the professionals' assessments on patient reported background information items (case-mix variables) and PRMs defined in ICHOM PCB set in terms of importance, and the appropriateness of the timing of the questionnaires. Also, key comments mentioned by at least $25 \%$ of the participants are reported. Generally speaking, participants felt that patient reported background information and the PROMs and PREMs included in ICHOM PCB set are important for delivering and developing maternity care in Finland. Compared with PROMs, more participants agreed on the importance of PREMs. However, most participants criticized the timing of data collection suggested in the 
ICHOM PCB set, which was deemed inappropriate for the Finnish maternity care pathway. A majority of participants considered "birth" (T3) as a problematic time point for data collection, mainly because that it is almost impossible for women who are suffering pain and discomfort during labor to answer PRMs questions and that it would be burdensome for medical staff who are already busy with taking care of labor to execute PRMs data collection. According to some participants, for some measures, e.g. motherinfant attachment and breastfeeding success, it is too early and pointless to inquire about at birth. Some mentioned that in Finland the last maternal checkup is at two to four months postpartum (slight difference exists between different healthcare regions), thus it would be more valuable to collect PRMs data during two to four months postpartum before the last maternal checkup instead of the evaluation at six months postpartum (T5) that might require extra service to be arranged for the mother. Participants noticed that most PROMs and PREMs data had not yet been routinely or systematically collected in Finland, while some PROMs and PREMs related questions had been asked from women via surveys or oral communication at Neuvola and delivery hospitals. Participants agreed that even though ICHOM uses internationally validated instruments in the standard sets, the instruments would need to be tailored for the Finnish population and maternity service context. The major adjustments reported by participants included 1) removing the questions on race, ethnicity and nationality, and replacing it with language or birth place instead, 2) adding health behavior related questions, e.g. asking women about their eating, alcohol consumption, and smoking, and e.g. domestic violence, 3) reframing and simplifying the questions on confidence with breastfeeding, 4) removing "water" from the feeding options, since in Finland it is not recommended to give water to infants less than six months old, 5) adding "solid food" to feeding options, 6) using Edinburgh Postnatal Depression Scale (EPDS) as the mental health screening tool during pregnancy, 7) identifying the type of professional, service provider, and service context when asking questions on satisfaction, decision-making, and confidence in healthcare providers, 8) asking questions on satisfaction with care instead of satisfaction with the result of care, 9) removing the item "The delivery room was clean and hygienic" from the questions on birth experience, since cleanliness is not a problem in Finland, and clarifying the item "I came through childbirth virtually unscathed", 10) redesigning the time points of data collection for each measure. In addition, six participants suggested to add a patient reported measure of fear of childbirth (FOC), an issue that has gained a special attention in Finland. According to the professionals we interviewed, the incidence of FOC in Finland has been increasing recently, affecting $6 \%-10 \%$ of pregnant women. Some participants also suggested that questions for partners should be included as well, since they play important roles during the pregnancy and in raising the child.

\section{Viewpoints on implementation of PRMs}

Table 3 displays professionals' expectations and concerns on the implementation of PRMs in Finnish maternity care. They are organized according to the modified UTAUT framework under five primary themes, i.e. Performance, Effort, Condition, Risk, Social norms and influence, and grouped into inductively developed sub-themes. For each sub-theme, we counted the number of professionals who mentioned it to evaluate the importance of each sub-theme compared to other. Table 3 also presents sample quotations. 
Performance. Over half of our participants mentioned that implementing PRMs would bring advantages to the overall maternity care system by improving the maternity care pathway, building an integrated and continuous care system, and by enabling national and international comparisons. The same number of participants felt that through PRMs providers would get valuable information on the patients' perspective and deepen their understanding of patients and their health status. Half of the interviewees emphasized the managerial benefits, including getting a real picture of the overall situation, identifying the deficiencies in the service system, prioritizing the key issues to be addressed, and allocating resources better. Many participants mentioned that PRMs approach would improve health service practices and increase patient satisfaction.

Effort. There was a consensus among participants that it would be not easy to collect and process PRM data. Participants stated that a lot of effort should be put into motivating women, partners, and personnel, into providing training, into developing advanced data processing tools and systems, and into administering data access and protecting the data. Fourteen participants suggested that it would be important to develop contextually appropriate measures, which should be understandable and easy for women to respond to and invite the partner to express their feelings and views. Another important aspect mentioned by nine participants was that the data should be properly utilized, and the data should be translated into actions. Some participants pointed out that it was necessary to integrate PRMs into current information systems and into current routine practices. A few of participants realized that it was necessary to establish closer collaboration between different professionals, and between different providers, and to optimize information sharing.

Condition. Participants identified both facilitators and obstacles for implementing PRMs in Finnish maternity care. Over half of participants agreed that current culture in the maternity care services in Finland and current trends, e.g. focusing on service experiences and promoting women's active role in the care process, would support implementation of PRMs. Eight participants believed that the new hospital information system, Apotti (a regionally uniform social and healthcare information system, integrating all health and social care data, and allowing patients to check medical information, report health status and communicate with professionals) [36], to be implemented in HUS hospitals as well as in the primary care in the municipalities in the hospital district would make the collection of ePROMs (electronic PROMs) and ePREMs (electronic PREMs) feasible. The commonly mentioned obstacles for the implementation of PRMs included the following. First, the busy and exhausted mothers might not be motived to give answers to excessive and repeated questions during the care pathway. Second, busy staff would view PRMs as extra work and refused to change their daily routines. Third, in the districts without wellestablished integration between primary care and secondary care, the disconnection and inconsistency between different providers would make the data collection and transferring challenging.

Perceived risk. More than half of professionals noted that there was a high risk of data bias caused by women refusing to answer excessive and repeated questions, dropping out from answering the PRM questionnaires during the care pathway, or being left out due to language barriers or other factors. Other 
risks mentioned by some professionals included not utilizing the collected data properly and causing an extra burden to the staff.

Social norms and influence. Our participants could not identify someone, who could influence their decision to use PRMs. However, they acknowledged that PRMs are promoted by current trends in healthcare, including patient centered care, healthcare equality, integrated and continuous care, and value-based healthcare.

\section{Discussion}

\section{Main contributions}

This is the first study from Finland, even the first one from Nordic countries, to explore the applicability of PROMs and PREMs defined by ICHOM for maternity care. This study adds valuable insights into the value, acceptability and feasibility of applying PRMs in the routine practices of maternity services. Specifically, this study observes the acceptability of the emerging potential international standard among highly qualified professionals who have not participated in developing the standard. With this study, we gain a preliminary but important view of Finnish professionals' expectations on the development and implementation of PRMs for improving public maternity care, which lay a ground for further study and implementation of PRMs. While this study can serve as an example for other Nordic countries with similar social contexts and maternity service culture to develop and implement PRMs, it can also help to address the worldwide shortage on the understanding of applying PRMs for developing patient-centered and value-based health care.

\section{Interpretation}

The findings of the current study indicate that the PCB set that has not been implemented in Finland yet contains PROMs, PREMs and other patient reported information (i.e. case-mix variables), which are important and useful in delivering and developing maternity care. Similar results have been reported earlier from Netherlands [23, 24]. In particular, PREMs which cover issues like patient's active role, birth experience, and satisfaction [30] were rated by almost all participants as being "important". This finding is in accordance with the observations made by Laureij et al (2019) from Netherlands [3]. However, the contents of patient reported measures need to be customized and adapted to Finnish culture and maternity care service setting. In contrast to findings in Laureij et al (2019), according to which the recommended timing of the data collection was appropriate [24], our study suggests that the time points of data collection should be adapted to fit into the local service pathway. In addition, due to the changes of physical and mental status during pregnancy and childbirth, women's capability to answer PRMs questions could be affected. This should be considered in the selection of time points of data collection. Particularly, consistent with Depla et al (2020) [23], our study indicates that collecting data during or just after birth is problematic, causing burdens on women and staff. Instead of collecting PRMs data at six 
months after the birth, collection sometime between two to four months postpartum would be more practical, as this is the time frame for the final maternal checkup offered in Finland. While changes are inevitable, it is worth noting that modifying the recommended questions, some of which are internationally validated scales, and adjusting the time flow will make international comparisons weaker and compromise the advantage of using standard PROMs and PREMs for cross-country benchmarking. Trade-offs have to be made in the development and implementation of the PRMs.

The potential value of PRMs for information augmentation, service and care improvement, managerial improvements, and maternity care system development was recognized by Finnish healthcare professionals and researchers. Our interviewees felt that introducing PRMs into maternity care was strongly recommended. They reported that patient-centered care, healthcare equality, integrated and continuous care, evidence-based medicine and value-based healthcare are the main healthcare principles and trends driving the introduction of PRMs. Professionals also recognized that implementation of PRMs would support current efforts to build a patient-centered and value-based service culture in Finland. They also indicated that current advanced IT infrastructure, including the new information system, Apotti, used in the capital region, would help to make ePROMs and ePREMs collection, processing and management possible. Although it has been regarded as an important, and feasible goal, implementing PRMs in the Finnish maternity care has challenges and risks. For a successful implementation, various steps like developing suitable questions, redesigning the service pathway and protocols, and motivating patients were considered important. Our interviewees particularly criticized the fragmented service system, where the main providers, i.e. Neuvola and hospitals, have not been collaborating well enough, and information sharing and transfer has not been effectively organized. But according to most of professionals, the implementation of PRMs would facilitate the process of integration. Delivering integrated and continuous care has been an important goal announced by the Finnish authorities [27].

\section{Practical implication}

This study describes Finnish professionals' viewpoints on introducing PRMs and adapting ICHOM PCB patient reported measures into Finnish maternity care. It provides important strategic and practical insights for planning and conducting implementation pilot(s) in the country. The key suggestions derived from this study for preparing a future implementation pilot, which could also be considered for implementing PRMs in other similar contexts include

- Developing a minimum set of patient-reported measures and items, which covers the most relevant and important issues and contains generic, context/condition/disease-specific questions, avoids unethical questions (e.g. race and sexual activity for certain groups), involves the partners' view, and has data collection time points that align with the local service pathway;

- Integrating patient-reported data into current information systems, merging the patient-reported outcomes and experiences data with clinician-reported data and other medical data, and building an intelligent system to analyze the data and visualize the results; 
- Establishing favorable collaboration of multiple stakeholders, identifying a coordinating group for daily operations, and defining roles, responsibilities, and data access for each stakeholder;

- Reforming service process and protocol to integrate PRMs data collection and utilization as part of care, setting sessions to review patient reported data and discuss emerging issues with patients, and organizing necessary follow-ups for health issues revealed by PRMs;

- Motivating women and partners to respond to PRMs questions by informing them of the added value of PRMs, and developing user-friendly digital tools;

- Training users of PRMs data, educating front-line workers to respond to the issues raised through PROM questionnaires, and appointing a task force to respond to issues arising from PREMs data

\section{Limitation And Future Studies}

With a limited number of interviews and a limited geographical coverage, the results of this qualitative study may not be generalizable to all Finnish hospitals or to professionals in other countries. For the next step, we will conduct comprehensive surveys among professionals in different hospital districts to get a more confirmative and generalizable view on the applicability of PRMs in Finnish maternity care. Another limitation of our study is the lack of views from patients, i.e. women, partners and families, who are the key stakeholders in the maternity care network. Before implementing PRMs of maternity care, we will conduct interviews and surveys with women and their partners and investigate their views regarding PRMs. In addition, context specificity might limit the scope of the findings, as e.g. culture and IT infrastructure can have a large impact on the professionals' views on the importance and feasibility of PRMs collection and utilization. As the project progresses, we will also compare our results with those of similar projects around the world and draw more generalizable conclusions, so as to provide contexttransferable solutions of PRMs implementation.

\section{Conclusion}

This study reveals that professionals working for Finnish public maternity care consider the PROMs and PREMs and other patient reported items recommended by ICHOM to be relevant and important for delivering and developing maternity service in Finland, and see the need to introduce PRMs into Finnish public maternity care system with the promise to improve maternity care. But the implementation of PRMs will require a lot of efforts, e.g. developing suitable questions, redesigning service pathway and protocols, and motivating patients considered important.

\section{List Of Abbreviations}

- PRMs: Patient Reported Measures

- PROMs: Patient Reported Outcome Measures

- PREMs: Patient Reported Experience Measures

- ICHOM: International Consortium on Health Outcome Measurement 
- PCB set: an outcomes and quality measurement set for pregnancy and childbirth developed by ICHOM

- THL: National Institute for Health and Welfare of Finland

- PHN: Public Health Nurses

- UTAUT: Unified Theory of Acceptance and Use of Technology

- HUS: Helsinki and Uusimaa Hospital District

- ePROMs: electronic Patient Reported Outcome Measures

- ePREMs: electronic Patient Reported Experience Measures

- EPDS: Edinburgh Postnatal Depression Scale

- FOC: Fear of Childbirth

\section{Declarations}

\section{Ethics approval and consent to participate}

This non-medical study involves human participants (i.e. healthcare professionals as interview participants), and it follows the principles and guidelines defined in the Declaration of Helsinki. The main ethical considerations regarding this study included: (i) professionals were informed on the study's nature and the interview protocol; (ii) professionals voluntarily participated in the interviews and gave informed consent in oral or written; (iii) professionals had a right to withdraw at any time knowing it would not cause any harm to them or affect them in any way; and (iv) no information revealing the informant's identity would be publicized. This study got ethics approval and research permission from Vantaa Social and Health Care Service (ID: VD/9096/13.00.00/2019)

\section{Consent to publication}

All identifying information has been removed within this publication, thus consent for publication is not applicable.

\section{Availability of data and materials}

The dataset generated and analysed for this study is not publicly available due to the restrictions claimed in the research permissions and letter to interviewees. Data are however available from the authors upon reasonable request and with permissions of ethical committees of corresponding hospital districts and municipalities. For requesting the access to data and concerning issues related to the data, please contact with the corresponding author.

\section{Competing interests}

Not applicable 
This study is financially supported by Finland PoDoCo Fundation of Economic Education.

\section{Authors' Contributions}

The first author of this paper, Chen, was responsible for defining the topic, designing the study, and implementing the research. She designed the interview questions, collected and analysed the data, interpreted the results, and drafted the paper. Väyrynen participated in analysing the data, interpreting the findings, and revising the paper throughout the drafting process. Leskelä guided the empirical research, provided research expert comments, revised the paper often throughout the drafting process. Tekay, Heinonen and Lillrank provided valuable insights into the research plan, gave advices on data collection and interpretation, and improved the manuscript. Torkki gave advice for the study's design, data analysis, and interpretation of the results, supervised the whole research and gave the final approval of the version to be published. All authors participated sufficiently in the work to take public responsibility for appropriate portions of the content, and agreed to be accountable for all aspects of the work in ensuring that questions related to the accuracy or integrity of any part of the work were appropriately investigated and resolved. The development of the paper for publication was the collective effort of all the authors.

\section{Acknowledgements}

The work reported here was highly supported by different hospital districts and municipalities in Finland. We thank all participating professionals and experts for their efforts. We acknowledge Ms. Rosmarie Kallankari from Nordic Healthcare Group Oy for helping in interviews and data transcription. Gratitude is owed to all who made this study and paper possible.

\section{References}

1. Berwick D, Black N, Cullen D, Deerberg-Wittram J, Degos L, Diverty B, et al. Recommendations to OECD ministers of health from the high level reflection group on the future of health statistics: Strengthening the international comparison of health system performance through patient-reported indicators. OECD.org; 2017. Available from: https://www.oecd.org/els/health-

systems/Recommendations-from-high-level-reflection-group-on-the-future-of-health-statistics.pdf

2. Fung $\mathrm{CH}$, Hays RD. Prospects and challenges in using patient-reported outcomes in clinical practice. Qual Life Res. 2008;17(10):1297-302.

3. Tew M, Dalziel K, Clarke P, Smith A, Choong PF, Dowsey M. Patient-reported outcome measures (PROMs): can they be used to guide patient-centered care and optimize outcomes in total knee replacement? Qual Life Res. 2020;10.1007/s11136-020-02577-4.

4. Damman OC, Jani A, de Jong BA, Becker A, Metz MJ, de Bruijne MC, et al. The use of PROMs and shared decision-making in medical encounters with patients: An opportunity to deliver value-based health care to patients. J Eval Clin Pract. 2020;26(2):524-40.

5. Black N, Varaganum M, Hutchings A. Relationship between patient reported experience (PREMs) and patient reported outcomes (PROMs) in elective surgery. BMJ Qual Saf. 2014;23(7):534-42. 
6. Kingsley C, Patel S. Patient-reported outcome measures and patient-reported experience measures. BJA Education. 2017;17(4):137-44.

7. Porter ME. What is value in health care? N Engl J Med. 2010;363(26):2477-81.

8. Porter ME, Teisberg EO. Redefining competition in health care. Harv Bus Rev. 2004:64-77.

9. Dawson J, Doll H, Fitzpatrick R, Jenkinson C, Carr AJ. The routine use of patient reported outcome measures in healthcare settings. BMJ. 2010;340:c186.

10. Chen J, Ou L, Hollis SJ. A systematic review of the impact of routine collection of patient reported outcome measures on patients, providers and health organisations in an oncologic setting. BMC Health Serv Res. 2013;13(1):211.

11. Yang LY, Manhas DS, Howard AF, Olson R. Patient-reported outcome use in oncology: a systematic review of the impact on patient-clinician communication. Support Care Cancer. 2018;26(1):41-60.

12. Nelson EC, Eftimovska E, Lind C, Hager A, Wasson JH, Lindblad S. Patient reported outcome measures in practice. BMJ. 2015;350:g7818.

13. Geller PA. Pregnancy as a stressful life event. CNS spectrums. 2004;9(3):188-97.

14. Delgado A, de Oliveira PdNF, de Góes PSA, Lemos A. Development and analysis of measurement properties of the "maternal perception of childbirth fatigue questionnaire"(MCFQ). Brazilian journal of physical therapy. 2019;23(2):125-31.

15. Symon A, Downe S, Finlayson KW, Knapp R, Diggle P. The feasibility and acceptability of using the Mother-Generated Index (MGI) as a Patient Reported Outcome Measure in a randomised controlled trial of maternity care. BMC Med Res Methodol. 2015;15(1).

16. Brédart A, Marrel A, Abetz-Webb L, Lasch K, Acquadro C. Interviewing to develop Patient-Reported Outcome (PRO) measures for clinical research: eliciting patients' experience. Health and quality of life outcomes. 2014;12(1):15.

17. Dickinson F, McCauley M, Smith H, Van Den Broek N. Patient reported outcome measures for use in pregnancy and childbirth: A systematic review. BMC Pregnancy Childbirth. 2019;19(1):155-62.

18. Gregory KD, Korst LM, Saeb S, McCulloch J, Greene N, Fink A, et al. Childbirth-specific patient-reported outcomes as predictors of hospital satisfaction. Am J Obstet Gynecol. 2019;220(2):201.e1-.e19.

19. Mahmud A, Morris E, Johnson S, Ismail KM. Developing core patient-reported outcomes in maternity: PRO-Maternity. BJOG : an international journal of obstetrics and gynaecology. 2014;121(Suppl 4):159.

20. International Consortium for Health Outcomes Measurement (ICHOM) [cited 22 October 2020]. Available from: http://www.ichom.org/].

21. Nijagal MA, Wissig S, Stowell C, Olson E, Amer-Wahlin I, Bonsel G, et al. Standardized outcome measures for pregnancy and childbirth, an ICHOM proposal. BMC Health Serv Res. 2018;18(1):1-12.

22. International Consortium for Health Outcomes Measurement (ICHOM) - The ICHOM Standard Set for Pregnancy And Childbirth [cited 23 October 2020]. Available from: https://www.ichom.org/portfolio/pregnancy-and-childbirth/]. 
23. Depla AL, Ernst-Smelt HE, Poels M, Crombag NM, Franx A, Bekker MN. A feasibility study of implementing a patient-centered outcome set for pregnancy and childbirth. Health science reports. 2020;3(3):e168.

24. Laureij LT, Been JV, Lugtenberg M, Ernst-Smelt HE, Franx A, Hazelzet JA, et al. Exploring the applicability of the pregnancy and childbirth outcome set: A mixed methods study. Patient Educ Couns. 2019;103(3):642-51.

25. Al-Shammari I, Roa L, Yorlets RR, Akerman C, Dekker A, Kelley T, et al. Implementation of an international standardized set of outcome indicators in pregnancy and childbirth in Kenya: Utilizing mobile technology to collect patient-reported outcomes. PLoS One. 2019;14(10):e0222978.

26. Rutherford C, Campbell R, Tinsley M, Speerin R, Soars L, Butcher A, et al. Implementing PatientReported Outcome Measures into Clinical Practice Across NSW: Mixed Methods Evaluation of the First Year. Applied Research in Quality of Life. 2020;10.1007/s11482-020-09817-2

27. Wrede S, Benoit C, Einarsdottir T. Equity and dignity in maternity care provision in Canada, Finland and Iceland. Can J Public Health. 2008;99(2):16-21.

28. Hägg MM. Effects of Maternity Ward Closures on Maternal Health in Finland [Master thesis]. University of Eastern Finland2020.Available from: https://epublications.uef.fi/pub/urn_nbn_fi_uef20200932/urn_nbn_fi_uef-20200932.pdf

29. Gissler M, Kiuru S. Perinatal statistics - parturients, delivers and newborns 2018 (Perinataalitilasto: synnyttäjät, synnytykset ja vastasyntyneet 2018). National Institute for Health and Welfare of Finland (Terveyden ja hyvinvoinnin laitos, THL); 2019. Available from: http://www.julkari.fi/bitstream/handle/10024/138998/Tr49_19.pdf?sequence=5\&isAllowed=y

30. Kortet S, Melender H-L, Klemetti R, Kääriäinen M, Kaakinen P. Mothers' perceptions of the quality of maternity services at Finnish maternity units: A cross-sectional study. Nordic Journal of Nursing Research. 2020;10.1177/2057158520937541.

31. Venkatesh V, Thong JY, Xu X. Unified theory of acceptance and use of technology: A synthesis and the road ahead. Journal of the Association for Information Systems. 2016;17(5):328-76.

32. Guest G, Bunce A, Johnson L. How many interviews are enough? An experiment with data saturation and variability. Field methods. 2006;18(1):59-82.

33. Marshall MN. Sampling for qualitative research. Fam Pract. 1996;13(6):522-6.

34. Azungah T. Qualitative research: deductive and inductive approaches to data analysis. Qualitative Research Journal. 2018;18(4):383-400.

35. Smith J, Firth J. Qualitative data analysis: the framework approach. Nurse Res. 2011;18(2):52-62.

36. Apotti official website [Available from: https://www.apotti.fi/].

\section{Tables}

Table 1. Basic characteristics of participants 


\begin{tabular}{|ll|}
\hline Characteristics of participants & Overall (N=20) \\
\hline Gender & $2(10 \%)$ \\
\hline Male & $18(90 \%)$ \\
\hline Female & \\
\hline Work experience (years of working in the area of pregnancy and childbirth) & \\
\hline Mean & 21.2 \\
\hline Max & 40 \\
\hline Min & 2 \\
\hline Current occupation (one interviewee may have more than one positions) & \\
\hline Gynecologist \& Obstetrician & 7 \\
\hline Midwife & 5 \\
\hline Public Nurses & 2 \\
\hline Manager/administrator & 9 \\
\hline Educator/trainer & 3 \\
\hline Researcher & 4 \\
\hline Organization (one interviewee may work for more than one organizations) & \\
\hline Delivery hospital & 10 \\
\hline Neuvola & 5 \\
\hline Midwifery school & 2 \\
\hline THL & 4 \\
\hline Region & \\
\hline Capital region & \\
\hline Other regions & \\
\hline & \\
\hline
\end{tabular}

Table 2. Assessing patient reported case-mix variables and patient reported measures defined in ICHOM PCB Set (Interviewee No.= 20) 


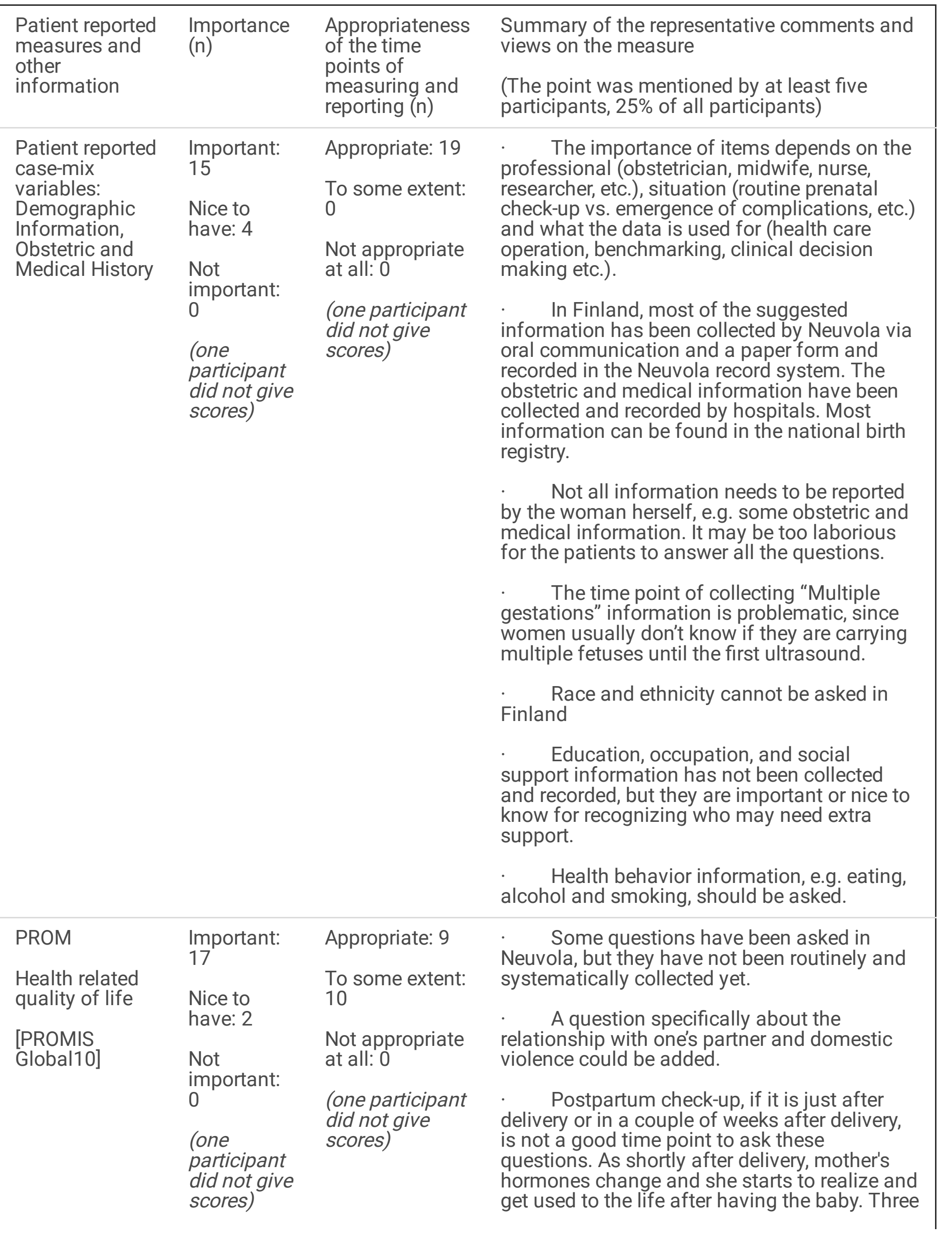


months postpartum could be a good time in Finland to measure women's quality of life after having a baby.

\begin{tabular}{|c|c|c|}
\hline PROM & Important: & Appropriate: 6 \\
\hline Incontinence & & To some \\
\hline [ICIQ-SF] & $\begin{array}{l}\text { Nice to } \\
\text { have: } 5\end{array}$ & extend:13 \\
\hline [Wexner] & Nht & Not appropriate \\
\hline & $\begin{array}{l}\text { Not } \\
\text { important: } \\
0\end{array}$ & $\begin{array}{l}\text { at all: } 0 \\
\text { (one participant } \\
\text { did not give }\end{array}$ \\
\hline & $\begin{array}{l}\text { (one } \\
\text { participant } \\
\text { did not give } \\
\text { scores) }\end{array}$ & scores) \\
\hline
\end{tabular}

- Some descriptions are quite vague, e.g. large amount, little amount, etc.
- In Finland, the information has not been routinely or systematically collected yet.

At the postpartum checkup, professionals may check, observe and record it, but it is not regularly reported by women.

\section{- Incontinence during pregnancy and} childbirth is a very rare problem in Finland. It could be used as a targeted measure for those women who have incontinence problems either during pregnancy or after delivery.
- $\quad$ Postpartum check-up, the period defined by ICHOM, is a quite early point to ask incontinence related questions. The most important time point is three to six months postpartum, when treatment is necessary if women still have incontinence.

\begin{tabular}{|c|c|c|}
\hline PROM & $\begin{array}{l}\text { Important: } \\
16\end{array}$ & Appropriate: 7 \\
\hline $\begin{array}{l}\text { Pain with } \\
\text { intercourse }\end{array}$ & $\begin{array}{l}\text { Nice to } \\
\text { have: } 4\end{array}$ & $\begin{array}{l}\text { To some } \\
\text { extent: } 13\end{array}$ \\
\hline $\begin{array}{l}{[\text { PROMIS }} \\
\text { SFFAC102] }\end{array}$ & $\begin{array}{l}\text { Not } \\
\text { important: } \\
0\end{array}$ & $\begin{array}{l}\text { Not appropriate } \\
\text { at all: } 0\end{array}$ \\
\hline
\end{tabular}

- In Finland, the data has not been systematically or routinely collected yet. Intercourse issues are discussed if women bring them up.

Not appropriate bring them up.

- It might be a challenging theme for some ethnic minorities or someone concerning privacy

- At the first postpartum check-up, the time point defined by ICHOM, women are not usually having intercourse yet. The best time to measure this in Finland is three to four months after the delivery.

PROM

Confidence with

role as a

mother

[ROLECONF]
Important: 18

Nice to

have: 1

Not important: 0

(one participant did not give scores)
PROM Important: Appropriate: 5
Appropriate: 8

To some extent: 11

Not appropriate at all: 0

(one participant did not give scores)
Mother's confidence has not been routinely or systematically measured in Finland.

It is too early to measure mother's confidence at the beginning of pregnancy; It is more logical to ask about it at the end of the pregnancy. 


\begin{tabular}{|c|c|c|}
\hline $\begin{array}{l}\text { Mother-infant } \\
\text { attachment }\end{array}$ & $\begin{array}{l}\text { Nice to } \\
\text { have: } 1\end{array}$ & $\begin{array}{l}\text { To some extent: } \\
14\end{array}$ \\
\hline [MIBS] & $\begin{array}{l}\text { Not } \\
\text { important: } \\
0 \\
\text { (one } \\
\text { participant } \\
\text { did not give } \\
\text { scores) }\end{array}$ & $\begin{array}{l}\text { Not appropriate } \\
\text { at all: } 0 \\
\text { (one participant } \\
\text { did not give } \\
\text { scores) }\end{array}$ \\
\hline PROM & Important: & Appropriate: 8 \\
\hline $\begin{array}{l}\text { Maternal } \\
\text { confidence with } \\
\text { breastfeeding }\end{array}$ & $\begin{array}{l}\text { Nice to } \\
\text { have: } 2\end{array}$ & $\begin{array}{l}\text { To some extent } \\
9\end{array}$ \\
\hline $\begin{array}{l}\text { [BFINTENT] } \\
\text { [BFCONFID] } \\
\text { [BSES-SF] }\end{array}$ & $\begin{array}{l}\text { Not } \\
\text { important: } \\
0 \\
\text { (three } \\
\text { participants } \\
\text { did not give } \\
\text { scores) }\end{array}$ & $\begin{array}{l}\text { Not appropriate } \\
\text { at all: } 0 \\
\text { (three } \\
\text { participants did } \\
\text { not give scores) }\end{array}$ \\
\hline
\end{tabular}

- Feelings towards the baby could be very complicated and vary over time.

- It is impossible for women to answer the questions during or just after delivery.

A good moment to measure this in Finland is at two to four months postpartum 


\begin{tabular}{|c|c|c|c|}
\hline \multirow{4}{*}{$\begin{array}{l}\text { PROM } \\
\text { Postpartum } \\
\text { depression_Part } \\
1 \text { [PHQ-9] }\end{array}$} & \multirow{2}{*}{$\begin{array}{l}\text { Important: } \\
17 \\
\text { Nice to } \\
\text { have: } 1\end{array}$} & Appropriate: 12 & \multirow{2}{*}{$\begin{array}{l}\quad \text { Mental health of women during } \\
\text { pregnancy and childbirth has been a very } \\
\text { important issue, and depression is one of the } \\
\text { most important PROMs. }\end{array}$} \\
\hline & & $\begin{array}{l}\text { To some extent: } \\
7\end{array}$ & \\
\hline & \multirow{2}{*}{$\begin{array}{l}\text { Not } \\
\text { important: } \\
0 \\
\text { (two } \\
\text { participants } \\
\text { did not give } \\
\text { scores) }\end{array}$} & $\begin{array}{l}\text { Not appropriate } \\
\text { at all:0 }\end{array}$ & $\begin{array}{l}\text { PHQ-9 is not used in Finland, but EPDS } \\
\text { has been widely used as a screening tool. }\end{array}$ \\
\hline & & $\begin{array}{l}\text { (one participant } \\
\text { did not give } \\
\text { scores) }\end{array}$ & $\begin{array}{l}\text { During pregnancy, it is good to screen in } \\
\text { the beginning to get baseline information, but } \\
\text { the 3rd trimester is more important and } \\
\text { relevant. }\end{array}$ \\
\hline \multirow{3}{*}{$\begin{array}{l}\text { PROM } \\
\text { Postpartum } \\
\text { depression_Part }\end{array}$} & $\begin{array}{l}\text { Important: } \\
18\end{array}$ & Appropriate: 11 & \multirow{2}{*}{$\begin{array}{l}\quad \text { Neuvola is now regularly collecting } \\
\text { relevant information about depression, using } \\
\text { EPDS. }\end{array}$} \\
\hline & \multirow{2}{*}{$\begin{array}{l}\text { Nice to } \\
\text { have: } 0\end{array}$} & $\begin{array}{l}\text { To some extent: } \\
7\end{array}$ & \\
\hline & & \multirow{2}{*}{$\begin{array}{l}\text { Not appropriate } \\
\text { at all: } 0 \\
\text { (two } \\
\text { participants did } \\
\text { not give scores) }\end{array}$} & $\begin{array}{l}\text { EPDS could be used to screen depression } \\
\text { during pregnancy, not just postpartum. }\end{array}$ \\
\hline [EPDS] & $\begin{array}{l}\text { Not } \\
\text { important: } \\
0 \\
\text { (two } \\
\text { participants } \\
\text { did not give } \\
\text { scores) }\end{array}$ & & $\begin{array}{l}\quad \text { In Finland, women are asked about } \\
\text { depression at the two to four-month } \\
\text { postpartum checkup }\end{array}$ \\
\hline \multirow{3}{*}{$\begin{array}{l}\text { PREM } \\
\text { Satisfaction } \\
\text { with the result } \\
\text { of care }\end{array}$} & $\begin{array}{l}\text { Important: } \\
19\end{array}$ & Appropriate: 14 & \multirow{2}{*}{$\begin{array}{l}\quad \text { A survey about the delivery experience } \\
\text { and satisfaction has been routinely conducted } \\
\text { in delivery hospitals. }\end{array}$} \\
\hline & 1 & $\begin{array}{l}\text { To some extent: } \\
6\end{array}$ & \\
\hline & & Not appropriate & $\begin{array}{l}\text { It is tricky to ask about the result of care } \\
\text { that is affected by many factors. It is more }\end{array}$ \\
\hline \multirow[t]{3}{*}{ [CARESAT] } & $\begin{array}{l}\text { Not } \\
\text { important: } \\
0\end{array}$ & & $\begin{array}{l}\text { logical to ask about care process or to ask } \\
\text { about care in general, and then to figure out } \\
\text { why women are not satisfied and what needs } \\
\text { to be improved. }\end{array}$ \\
\hline & \multirow[t]{2}{*}{$\begin{array}{l}\text { (one } \\
\text { participant } \\
\text { did not give } \\
\text { scores) }\end{array}$} & & $\begin{array}{l}\quad \text { Different care providers e.g. Neuvola and } \\
\text { delivery hospitals, or different professionals, } \\
\text { e.g. PHNs, midwives, and doctors, should be } \\
\text { evaluated separately. }\end{array}$ \\
\hline & & & $\begin{array}{l}\text { Instead of six months postpartum, two to } \\
\text { four months postpartum is a more suitable } \\
\text { time in the Finnish context to collect data and } \\
\text { discuss service experiences with women. }\end{array}$ \\
\hline \multirow{4}{*}{$\begin{array}{l}\text { PREM } \\
\text { Confidence as } \\
\text { an active } \\
\text { participant in } \\
\text { healthcare } \\
\text { decisions }\end{array}$} & \multirow[t]{2}{*}{$\begin{array}{l}\text { Important: } \\
20\end{array}$} & Appropriate: 14 & \multirow{2}{*}{$\begin{array}{l}\text { Involving patients in decision-making is a } \\
\text { highly important topic that needs to be } \\
\text { focused on now. }\end{array}$} \\
\hline & & $\begin{array}{l}\text { To some extent: } \\
6\end{array}$ & \\
\hline & $\begin{array}{l}\text { Nice to } \\
\text { have: } 0\end{array}$ & & \multirow{2}{*}{$\begin{array}{l}\quad \text { Different care providers, e.g. Neuvola and } \\
\text { delivery hospitals, or different professionals, } \\
\text { e.g. PHNs, midwives, and doctors, should be } \\
\text { evaluated separately, and certain services, e.g. }\end{array}$} \\
\hline & $\begin{array}{l}\text { Not } \\
\text { important: }\end{array}$ & at all: 0 & \\
\hline
\end{tabular}


$[\mathrm{HCR}] \quad 0$

PREM

Confidence in healthcare providers

$[\mathrm{HCR}]$

Important:

19

Nice to

have: 1

Not important:

0 prenatal screening, should be specifically evaluated.

The key question should be, whether women get the information they feel they need. in Finland

It has not been systematically measured

Instead of six months postpartum, two to four months postpartum is a more suitable time in the Finnish context to collect data and discuss with women about service experiences.
Appropriate: 14

To some extent: 6

Not appropriate at all: 0 


\section{Primary themes $\begin{aligned} & \text { Sub-themes } \\ & \text { ( } n=\text { the number }\end{aligned}$ Sample quotations of professionals mentioning the sub-theme)}

\section{Performance \\ whether an individual believes /expects that using the system will help to attain gains (expected usefulness) \\ Maternity care system development (11): improving the whole maternity care chain, building an integrated care system and continuity of care, and enabling national and international comparison}

Information augmentation (11): getting additional valuable, and structured information from patients, and better understanding women and their needs
"The whole maternity care chain will get benefits from this, from primary care to specialized care, and it will help to build continuity of care" (Interviewee No.4, Educator and Researcher)
"I believe PROMs and PREMs will be helpful. I can get more information before the appointment, and the discussions with clients will be better. Now the thing is that if I don't ask them, women may not necessarily tell me the problems. (If women do not actively report), for some problems or risky issues, I may never know, or know them too later. If I know, I can help immediately. Now we don't have so much time always during the appointment, so it is good to know some issues beforehand. Then I can arrange the time more efficiently and avoid unnecessary questions during the appointment. If women report the problems before the appointment, I can give more information they need and spend more time in talking about the issues. And I can prepare better. I can ask right and important questions. I will know my clients better." (Interviewee No. $17, \mathrm{PHN})$

"We will know the patient's feelings about the care, how they think about our system, and what care is wanted and needed." (Interviewee No. 18, Gynecologist, Obstetrician and Manager)
Improving management (10): getting a real picture of the overall situation, identifying the deficiencies in the service system, prioritizing the key issues to be addressed, and allocating
"So as a manager, I will know ... what we should do more in order to improve the services and where we should pay more attention. I can see bottlenecks, rearrange resources, set the goals more realistically and logically, and guide the work to a right direction." (Interviewee No.10, Gynecologist, Obstetrician and Manager)

"We can well identify the group that needs more help." (Interviewee No. 19, Midwife and Manager) 
resources

better

Improvement

of clinical

practices (8):

improving the

service

practices and

quality of care,

and increasing

patient

satisfaction

\section{Effort}

whether an

individual

believes

lexpects that

there is any

ease/complexity

and cost

associated with

the use of the

system
Data collection, processing and management (19):

motivating and

training

women,

partners, and

personnel,

developing

advanced data

tools and

systems,

defining data

access and

ensuring data

security

Development

of measures

and

instruments

(14):

developing

relevant, women-friendly

and family-

oriented

measures,

forming

questions in

way, and

avoiding

complicated,

long questions

and lengthy

questionnaires
"Previously, the outcomes and quality are defined from the expert's view, and the women's and family's views are missing. It is not only important to ask about (women's feelings and perspectives), but also to add them and reply them so. It is a very valuable way to collect the information that you are not able to get from the current birth registers, and to follow the process and monitor the situation before something happens." (Interviewee No. 1, Researcher)

"We should encourage the patient to report and let them know that they have the right and place to report problems and give feedback. That should be a part of the care, not just for improving the service system in general but helping the patient to solve problems and improving their own health. Patients have to be aware that they will be treated better after they report problems." (Interviewee No. 4, Educator and Researcher)
"In Finland, we are concerned about domestic violence issue, and we may need to add questions about relationship with partner." (Interviewee No. 12, Gynecologist, Obstetrician and Manager)

"It is also necessary to ask the partner too, because nowadays partners are involving more and more. Mother and partner could discuss and make decisions together, if there are any problems. This is Finnish culture that the partner involves a lot in the (pregnancy and childbirth) process. We should develop family-oriented questions" (Interviewee No. 17, PHN)

"Fear is the issue to be included in the questions. Currently, we are working a lot to reduce women's fear of childbirth" (Interviewee No. 8, Midwife and Manager)

"The questionnaires have to be made as easy as possible. Long and complicated questions will exhaust women. Women will refuse to give answers to lengthy and complicated questionnaires" (Interviewee No. 20, Midwife and Trainer). 
Data utilization and translation

(9): properly

utilizing the

data and

analysis

results,

responding to

the emerging

issues,

providing

necessary

interventions,

exploring the

relation

between

PROMs, PREMs

and other

outcome

measures.

Integration (7):

integrating

PROMs and

PREMs into

current

information

system and

into routine

service, making

it as a part of

care,

integrating it

with on-going

surveys

Collaboration

and

coordination

(6):

establishing

collaboration

between

different

professionals

and providers,

optimizing

information

sharing

Condition

whether an

individual

believes

/expects that

there is any

condition, e.g.

organizational
"If the score (answers to PRM questions) shows a bad situation, we need to think of how to give help and what is the next step. We need to know where to prefer the patient. If we don't prepare next step or tool (for the emerging issues), it is meaningless to collect the data." (Interviewee No. 8, Midwife and Manager)

\begin{abstract}
"A good way to integrate PROMs and PREMs into the daily service is to collect the PROMs and PREMs before each appointment. At the appointment, medical staff can discuss the emerging problems with patients, and give help or suggestions." (Interviewee No. 1, Researcher)
\end{abstract}

"Making PROMs and PREMs data collection as a part of care, not as survey work, is the goal." (Interviewee No. 11, Manager)
Current service culture and relevant efforts supporting PRMs implementation (13)
"It is challenging for hospitals to collaborate with Neuvola and municipalities. Hospitals and Neuvola should know how to collaborate in information collection and sharing." (Interviewee No. 3, Researcher)
"Our postnatal ward midwives ask women about delivery experiences, but not exactly with the same questions recommended by ICHOM. They (delivery hospitals) ask how it (the delivery) goes in general. It is mainly for the mother to speak out about their experiences. The scores are recorded. There have been some scale tools used to measure the mother's experiences, e.g. VAS (Visual Analogue Scale)." (Interviewee No. 6, Midwife) 
culture and

technical

infrastructure,

existing to

support/hinder

the use of the

system

"I believe our staff have positive attitude towards PROMs and PREMs approach. We want to make our clients satisfied with the service" (Interviewee No. 19, Midwife and Manager)

Busy mother

(13)

"It is hard to do the survey in the delivery room. Women only stay two hours there after delivery, and there is so much to do already, checking the mother and the baby, going to shower, eating, and a lot of paperwork. After delivery, women are busy with the newborn and don't have enough time to answer too many questions." (Interviewee No. 5, Educator)

Busy staff (9)

"We are already quite struggling with basic things and daily routines here at this moment. We do get lots of feedback on breastfeeding from mothers, complaining about insufficient information." (Interviewee No. 7, Midwife)

Current

information infrastructure, systems and tools enabling data collection, processing and management (8)
"We have started to adopt Apotti system (a regionally uniform social and healthcare information system, integrating all health and social care data, and allowing patients to check medical information, report health status and communicate with professionals). The primary healthcare of Vantaa has already implemented this system. Maybe it is a tool to collect PROMs and PREMs data. If Apotti comes to hospital, hospital can collect the information." (Interviewee No. 6, Midwife)

"iPad is used in delivery unit to collect women's feedback. Women answer questions using iPad. If mothers have $C$ sections, they will be asked in postnatal ward using iPad. Midwives give iPad to women after delivery." (Interviewee No. 5, Educator)

Lack of

integration

between

different

providers and

regions (8)
"Delivery hospitals and Neuvola use different information system, so how to collect the information, which organizations collect which information, and how the information could be shared and used... all are challenging." (Interviewee No. 1, Researcher)

"In Finland, different health regions use different information systems to collect and record patient information." (Interviewee No. 12, Gynecologist, Obstetrician and Manager)

Perceived risk

whether an individual

perceives that

using the system takes a possibility that something unpleasant or unwelcome
"Questions should be asked at several times during the care pathway, but repeating answering the questions may cause lots of drop-out. That is the risk." (Interviewee No. 1, Researcher)

"If the questionnaires are too long, patients will be tired of answering questions and will refuse to answer questions" (Interviewee No. 4, Educator and Researcher) 
(harm or

damage) will

happen to the

current system

and status

"Some groups of women, e.g. with low education or disadvantaged social status, may don't want to respond. Our problem now is that we don't get enough answers from immigrants and other disadvantaged groups. First (reason) is the language, and second is about culture. People from some cultural backgrounds are less likely to give feedback." (Interviewee No. 11, Manager)

Improperly utilizing the data (4)

Causing burdens on staff (3)

\section{Social norms} and influence

whether an individual believes that there is any social norm associated with the use of the system; whether an individual perceives that important others believe he or she should / should not use the system
Healthcare principles and trends (16): patientcentered care, healthcare equality, integrated care and continuity of care, evidence-based medicine, and value-based healthcare, which are driving the application of PRMs
"If we don't plan next step or tool (to address emerged problems), it is meaningless and wasteful to collect the data." (Interviewee No. 8, Midwife and Manager)

"Our staff are too busy and burdensome to collect the data and we do lot of paper works now. We have to concentrate on key stuff." (Interviewee No. 5, Educator)

"It is important to collect longitudinal PROMs and PREMs data for delivering patient-centered care. Now we have providerorganizing care, not truly patient-centered care." (Interviewee No. 4, Researcher and Educator)

"We want to develop connected integrated care, where delivery hospitals and Neuvola collaborate and cooperate. For the patient, it is a whole service package." (Interviewee No. 11, Manager)

"Evidence-based healthcare and patient-centered care will drive the use and implementation of PROMs and PREMs here in Finland" (Interviewee No. 20, Midwife and Trainer)

\section{Figures}




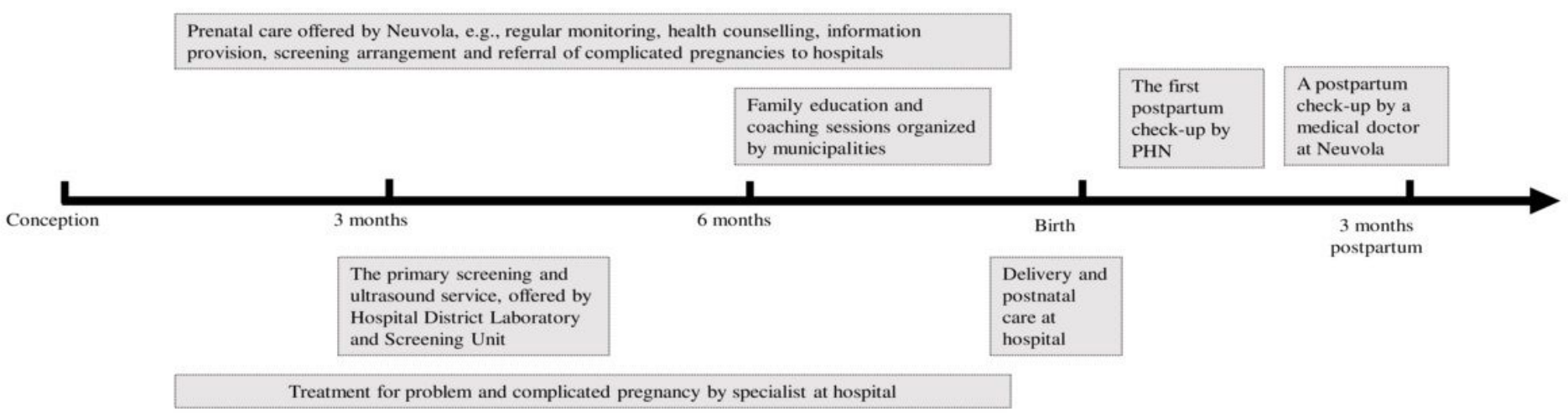

Figure 1

The general maternity care process in Finland

\section{Supplementary Files}

This is a list of supplementary files associated with this preprint. Click to download.

- Additionalfile1contentofpatientreportedinformationdefinedinICHOMPCBset.docx 